\title{
Article
}

\section{On-Chip Mid-Infrared Photothermal Spectroscopy using Suspended Silicon-on-Insulator Microring Resonators}

\author{
Anton Vasiliev, Aditya Malik, Muhammad Muneeb, Bart Kuyken, Roel Baets, and Gunther Roelkens
}

ACS Sens., Just Accepted Manuscript • Publication Date (Web): 07 Oct 2016

Downloaded from http://pubs.acs.org on October 7, 2016

\section{Just Accepted}

"Just Accepted" manuscripts have been peer-reviewed and accepted for publication. They are posted online prior to technical editing, formatting for publication and author proofing. The American Chemical Society provides "Just Accepted" as a free service to the research community to expedite the dissemination of scientific material as soon as possible after acceptance. "Just Accepted" manuscripts appear in full in PDF format accompanied by an HTML abstract. "Just Accepted" manuscripts have been fully peer reviewed, but should not be considered the official version of record. They are accessible to all readers and citable by the Digital Object Identifier (DOI®). "Just Accepted" is an optional service offered to authors. Therefore, the "Just Accepted" Web site may not include all articles that will be published in the journal. After a manuscript is technically edited and formatted, it will be removed from the "Just Accepted" Web site and published as an ASAP article. Note that technical editing may introduce minor changes to the manuscript text and/or graphics which could affect content, and all legal disclaimers and ethical guidelines that apply to the journal pertain. ACS cannot be held responsible for errors or consequences arising from the use of information contained in these "Just Accepted" manuscripts. 


\title{
On-Chip Mid-Infrared Photothermal
} Spectroscopy using Suspended

\section{Silicon-on-Insulator Microring Resonators}

\author{
Anton Vasiliev, ${ }^{*, \dagger, \ddagger}$ Aditya Malik, ${ }^{\dagger, \ddagger}$ Muhammad Muneeb, ${ }^{\dagger, \ddagger}$ Bart Kuyken, ${ }^{\dagger, \ddagger}$ Roel \\ Baets, ${ }^{\dagger, \ddagger}$ and Günther Roelkens $\nmid, \ddagger$ \\ $\dagger$ †hotonics Research Group, Ghent University-imec, Technologiepark-Zwijnaarde 15, 9052 \\ Ghent, Belgium \\ $\ddagger$ Center for Nano- and Biophotonics, Ghent University, Belgium \\ E-mail: Anton.Vasiliev@UGent.be
}

\begin{abstract}
Mid-infrared spectroscopic techniques rely on the specific "fingerprint" absorption lines of molecules in the mid-infrared band to detect the presence and concentration of these molecules. Despite being very sensitive and selective, bulky and expensive equipment such as cooled mid-infrared detectors are required for conventional systems. In this paper, we demonstrate a miniature CMOS-compatible Silicon-on-Insulator (SOI) photothermal transducer for mid-infrared spectroscopy which can potentially be made in high volumes and at a low cost. The optical absorption of an analyte in the midinfrared wavelength range $(3.25-3.6 \mu \mathrm{m})$ is thermally transduced to an optical transmission change of a micro-ring resonator through the thermo-optic effect in silicon. The photothermal signal is further enhanced by locally removing the silicon substrate beneath the transducer, hereby increasing the effective thermal isolation by a factor
\end{abstract}


of 40. As a proof-of-concept, the absorption spectrum of a polymer that has been locally patterned in the annular region of the resonator was recovered using photothermal spectroscopy. The spectrum is in good agreement with a benchmark Fourier-Transform Infrared spectroscopy (FTIR) measurement. A normalized noise equivalent absorption coefficient (NNEA) of $7.6 \times 10^{-6} \mathrm{~cm}^{-1} \mathrm{~W} / \mathrm{Hz}^{1 / 2}$ is estimated.

\section{Keywords}

Photothermal spectroscopy, mid-infrared, silicon photonics, microring resonator,gas sensing

Silicon photonics is emerging as an important platform for photonic integration. ${ }^{1}$ It is mostly being considered for telecom and datacom applications, but the portfolio of applications is now rapidly expanding towards miniaturized sensing systems such as label-free biosensing $^{2,3}$ and gas sensing. ${ }^{4,5}$

Spectroscopic sensors are an important class of devices that probe the unique "fingerprint" absorption spectrum of molecules. These sensors are unmatched in terms of selectivity for label-free detection of chemical components. The mid-infrared wavelength region is of particular interest due to the higher optical absorption cross-section at these frequencies as compared to telecom and visible wavelengths, because of the strong rovibrational resonances in this band. At the moment however, mid-infrared spectroscopic sensors are typically bulky and expensive. Considerable research is geared towards the integration and miniaturization of various optical components for this wavelength range. In particular for the 2 to $4 \mu \mathrm{m}$ wavelength range, a number of components have been demonstrated on the SOI platform such as filters, modulators, sources and detectors. ${ }^{6-11}$ However, the buried oxide buffer layer shows strong absorption for wavelengths beyond $4 \mu \mathrm{m} .{ }^{12}$ Integration of silicon photonics for spectroscopy is being considered on other substrate platforms such as $\mathrm{CaF}_{2}{ }^{13}$ as well as the use of chalcogenide glass waveguides. ${ }^{14-16}$

Photothermal spectroscopy is a highly sensitive and selective approach that measures the optical absorption of a material indirectly. The frequency dependent optical absorption $\alpha$ in- 
duces a change of the thermodynamic parameters: pressure, density and temperature which can be probed independently from the excitation source. ${ }^{17}$ These techniques have proven to be extremely sensitive and robust to environmental noise for the detection of various chemical compounds ${ }^{18-23}$ and microscopy applications. ${ }^{24-27}$ Limit of Detection (LOD) values of parts-per-trillion (ppt) have been demonstrated using quartz-enhanced photoacoustic spectroscopy (QEPAS) systems. ${ }^{28,29}$ One of the main reasons for the success of photothermal techniques is the fact that the signal is only originating from the absorption whereas in conventional transmission spectroscopy methods, scattering and reflection losses influence the measured signal. ${ }^{17}$ This makes these techniques particularly attractive for applications in the field. In this work, a novel photothermal spectroscopic transducer is demonstrated that uses a suspended microring resonator on a SOI chip to transduce the temperature change of a polymer analyte, induced by optical absorption in the mid-infrared wavelength range (3250-3600 nm or $\left.3075-2780 \mathrm{~cm}^{-1}\right)$.

The sensing principle is shown in Figure 1. The analyte is deposited (or captured in real-life applications) in the annular region of the microring resonator. The near-infrared (1550 nm) probe light transmission $\mathcal{T}$ coupled to the microring features a Lorentzian line shape near resonance where $m \lambda_{\text {probe,res }}=2 \pi R n_{\text {eff }}$ with $m$ the mode number, $R$ the ring waveguide radius and $n_{\text {eff }}$ the effective refractive index. The wavelength selective absorption of midinfrared radiation by the analyte gives rise to a wavelength selective temperature increase $\Delta T\left(\lambda_{\text {pump }}\right)$ which is transferred to the resonator. In turn, the increase in temperature causes a change of $n_{e f f}$. The change $\Delta n_{e f f}$ is read out by a near-infrared probe coupled to the ring resonator by measuring the shift in the transmission spectrum of the resonator. The probe wavelength $\lambda_{\text {probe }}$ is chosen at a point where the transmission spectrum has the highest slope $d \mathfrak{T} / d \lambda=\mathcal{T}^{\prime}$. By locking the probe laser to the slope of the optical resonance, the resonance shift is transduced to a power change $\Delta P_{\text {probe }}$ of the detected probe signal. By modulating the mid-infrared pump beam, the wavelength shift will translate into a modulated probe power change $\Delta P_{\text {probe }}\left(\lambda_{\text {pump }}\right)$ which can be read out sensitively using a lock-in type detection. 


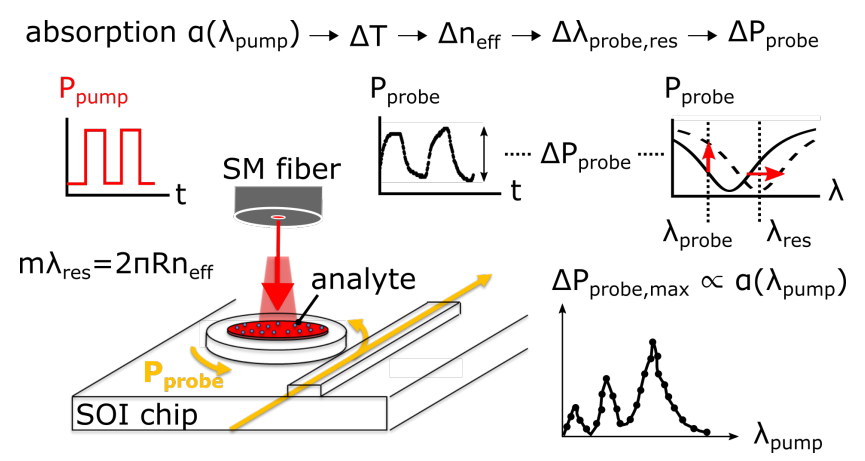

Figure 1: The heat generated through absorption of the mid-infrared pump beam increases the temperature of the ring resonator. Through the thermo-optic effect, the effective index of the waveguide changes, hereby changing the resonance wavelength $\lambda_{\text {res }}$ of the microring. For a fixed probe wavelength $\lambda_{\text {probe }}$, a change in probe power $\Delta P_{\text {probe }}$ is measured using a near-infrared detector. The absorption spectrum of the analyte can be reconstructed by scanning the pump wavelength and recording the maximum probe modulation $\Delta P_{\text {probe,max }}$.

\section{Experimental}

\section{fabrication}

The air-clad ring resonator devices were fabricated using a Multi Project Wafer service (MPW) in the $200 \mathrm{~mm}$ CMOS-pilot line at imec. ${ }^{30}$ The silicon device layer is $220 \mathrm{~nm}$ thick and rests on a $2 \mu \mathrm{m}$ thick buried thermal oxide (BOX). The TE-polarization grating couplers are defined using a $70 \mathrm{~nm}$ etch step. To achieve high-Q factor resonators, rib waveguides were used with an etch depth of $70 \mathrm{~nm}$ and $650 \mathrm{~nm}$ waveguide width. The ring resonators have a radius of $25 \mu \mathrm{m}$. A gap of $750 \mathrm{~nm}$ between the bus waveguide and ring resonator was chosen to obtain critical coupling. A photoresist AZ5214 from MicroChem was used as a mock-up analyte for the experiments. The resist was spin-coated and patterned in the annular region of the resonators using standard photolithography methods.

To increase the effective thermal resistance of the ring resonators, windows were opened in the silicon substrate by anisotropic etching in a KOH-solution. The samples were thinned 

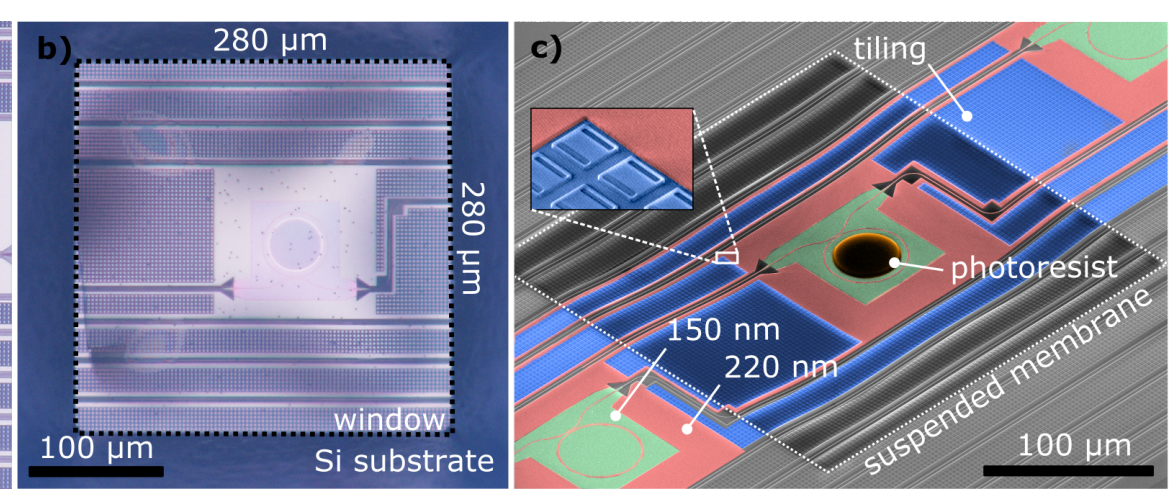

down from $700 \mu m$ to $150 \mu m$ thick and consecutively polished. A SiN/SiO $(400 / 500 \mathrm{~nm})$ thick hard mask was patterned on the backside of the samples. The samples were etched in a $\mathrm{KOH}$-solution $\left(80^{\circ} \mathrm{C} / 20 \%\right.$ concentration) for 180 minutes. A specially designed holder prevents the etchant from reaching the top device layer. The top device layer was additionally protected by using a photoresist layer to prevent spurious KOH-leakage from damaging the top surface. The opened windows are $280 \mu \mathrm{m}$ wide with an unintentional slight offset of $\sim 25 \mu \mathrm{m}$ from the center of the ring resonators, as seen in Figure 2.

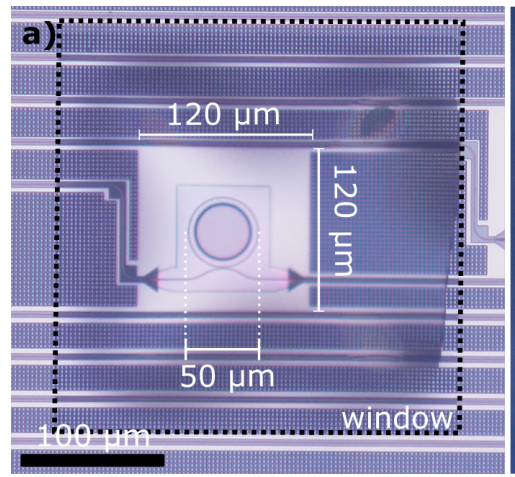

Figure 2: (a) Microscope image of suspended ring resonator with $\mathrm{R}=25 \mu \mathrm{m}$ and $1.35 \mu \mathrm{m}$ thick AZ5214 photoresist patterned in the annular region. (b) Backside image of the same device shows that a $280 \times 280 \mu m^{2}$ window was opened in the silicon substrate. The dark blue regions are the sidewalls of the anisotropically etched silicon substrate. (c) The various regions are indicated by partially coloring the tilted $\left(52^{\circ}\right)$ SEM image. The inset shows a detail of the tiling.

After substrate removal, the front side protection layer is removed in $\mathrm{O}_{2}$ plasma and a $1.35 \mu \mathrm{m}$ thick disk of AZ5214 is lithographically patterned on top of the suspended membrane resonators inside the annular region with a radius of $21 \mu \mathrm{m}$. The localization of the analyte within the ring waveguide has two reasons. The generated temperature change is localized near the ring and the optical transmission of the ring resonator is ideally unaltered. Additional losses induced by the analyte overlapping with the optical field would have a dramatic impact on the Q-factor of the ring and therefore the photothermal signal. The optical properties of the suspended microrings were practically unaltered by the processing as can be seen in Figure S1 and Figure S2 in the Supporting Information. 
Although no mechanical stability tests were performed on the devices, it is estimated through FEM simulation that the devices can withstand a mechanical shock that corresponds to a level 10 severity threshold according to the American National Standard S2.62-2009 which corresponds to an inelastic free fall from a height of $5 \mathrm{~m},{ }^{31}$ see also Figure $\mathrm{S} 3$ in the Supporting Information.

\section{measurement setup}

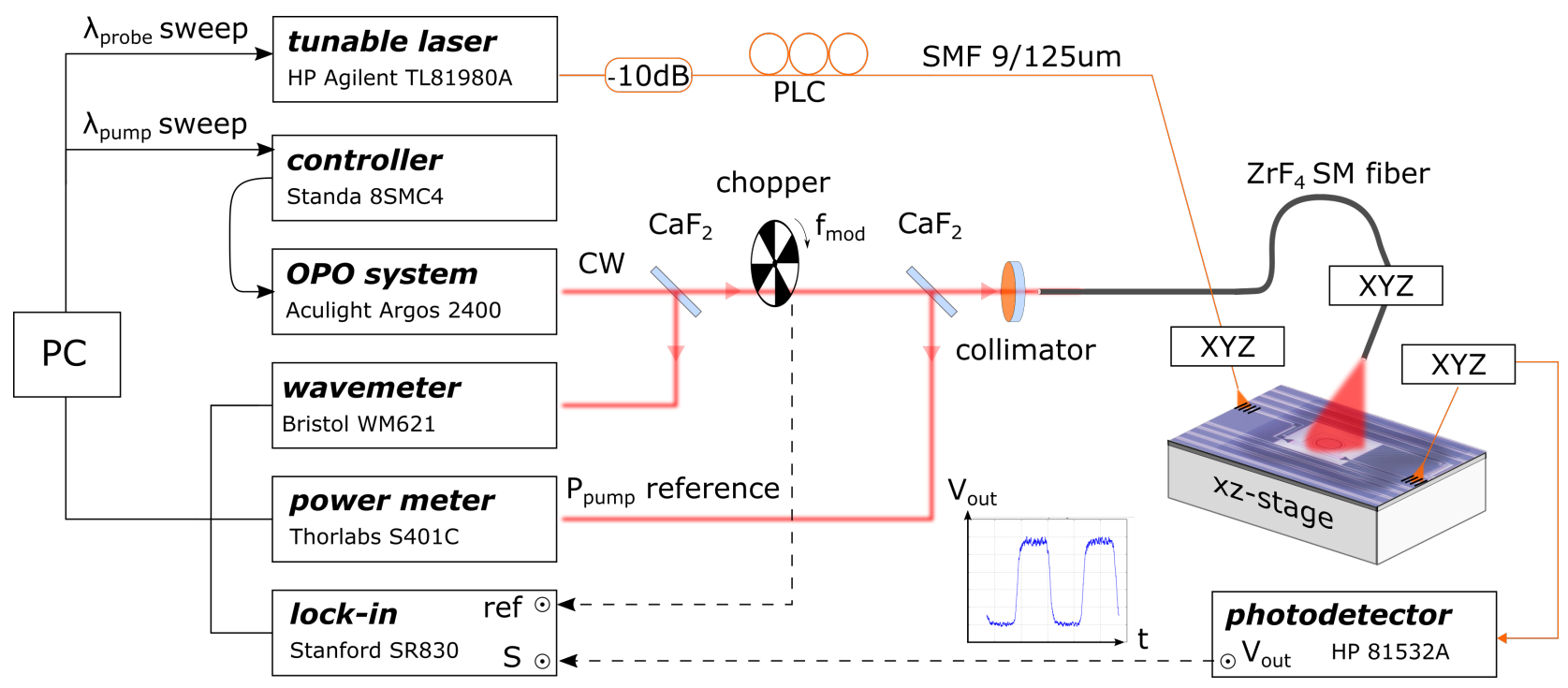

Figure 3: Schematic of the photothermal spectroscopy measurement setup. A tunable nearinfrared laser source at $1550 \mathrm{~nm}$ is vertically coupled to the ring resonator after passing a $10 \mathrm{~dB}$ attenuator and a polarization controller. The operating wavelength $\lambda_{\text {probe }}$ is set at the point with the highest slope of the ring resonator transmission spectrum. A freespace modulated tunable mid-infrared source (OPO) is step-scanned across the absorption spectrum 3250-3600 nm. The pump beam illuminates the analyte on the ring resonator from the end facet of a single mode $\mathrm{ZrF}_{4}$ fiber at an approximate distance of $100 \mu \mathrm{m}$ from the surface. At each $\lambda_{\text {pump }}$, the generated heat is transduced to a change in $\Delta n_{\text {eff }}$ and therefore the resonance wavelength of the ring. The resulting modulation of the probe $\Delta P_{\text {probe }}$ is readout using a lock-in amplifier at the modulation frequency. A fraction of the OPO power $P_{\text {pump }}$ is monitored in free-space using a thermopile power meter while the OPO wavelength is monitored by a wavemeter. The probe power $P_{\text {probe,max }}$ is monitored using the near-infrared photodetector at a wavelength off-resonance.

The measurement setup is schematically shown in Figure 3. An Optical Parametric Oscillator (OPO) system from Aculight was used as the mid-infrared pump source. The 
mid-infrared emission wavelength is swept by adjusting the intra-cavity etalon angle and the position of the periodically poled Lithium Niobate (PPLN) crystal. More details about the automation of the wavelength tuning process can be found here. ${ }^{32}$ The OPO light is focused into a $\mathrm{ZrF}_{4}$ single mode fiber.

A fraction of the OPO power is monitored in free-space using a thermopile detector S401C from Thorlabs. The output $\mathrm{ZrF}_{4}$ fiber end is cleaved and positioned $\sim 100 \mu \mathrm{m}$ above the ring resonator. Considering that the mode field diameter of the SM fiber is about $15 \mu m$ this ensures a beam spot on the resonator with a waist of $35 \mu \mathrm{m}$.

The tunable probe laser Agilent TL81980A is vertically coupled to the chip. The input power is attenuated by $10 \mathrm{~dB}$ in order to avoid nonlinear effects in the high Q ring resonator device. The on-chip probe power is estimated to be around $-23 \mathrm{dBm}$. A polarization controller is used to ensure that TE-polarized light is coupled to the chip. The analog output of the probe power meter HP81532A is coupled to a Stanford SR830 lock-in amplifier.

The mid-infrared pump wavelength is tuned from $3.25 \mu \mathrm{m}$ to $3.6 \mu \mathrm{m}$. Two different devices were measured with different parameters and resolution $\Delta \lambda_{\text {pump }}$, as seen in Figure 4 and table 1. To compensate for the slow ambient temperature change of the microring, the probe wavelength $\lambda_{\text {probe }}$ is re-optimized at each pump wavelength by searching for the local maximum of the photothermal signal using a step-wise hill-climbing algorithm, see also Figure S4 in the Supporting Information. The lock-in amplifier records 10 consecutive data points for each $\lambda_{\text {pump }}$ using an integration time of $100 \mathrm{~ms}$ and a filter slope of $18 \mathrm{~dB} /$ oct. The modulation frequency is $300 \mathrm{~Hz}$. The reference power levels are collected simultaneously. The acquired peak-to-peak modulation voltage amplitude $S$ at the modulation frequency is related to the device setup parameters through

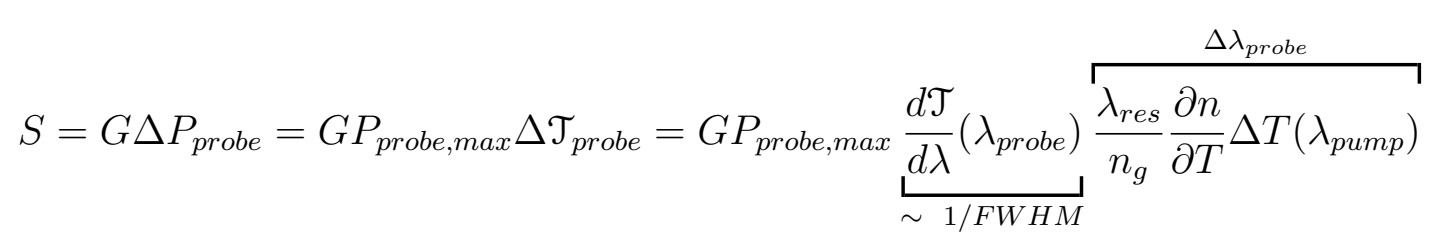


where $G$ is the gain of the photodetector, $\Delta P_{\text {probe }}$ is the change in transmitted probe power, $P_{\text {probe,max }}$ is the maximal transmitted probe power off-resonance, $\Delta \mathcal{T}_{\text {probe }}$ is the change in transmission, $d \mathcal{T} / d \lambda$ is the transmission slope of the ring resonator for a given $\lambda_{\text {probe }}, n_{g}$ is the group index, $\partial n / \partial T$ is the thermo-optic coefficient in silicon and $\Delta T\left(\lambda_{\text {pump }}\right)$ is the photothermally induced temperature modulation of the ring waveguide. The maximal slope is inversely proportional to the linewidth (FWHM) of the resonance. The FWHM of the microrings is in turn equal to $\lambda_{\text {probe,res }} / Q$ with $Q$ the loaded quality factor of the resonator. ${ }^{33}$ The relationship between the temperature increase $\Delta T$ and microring resonance shift $\Delta \lambda_{\text {probe }}$ has been used in equation $1 .{ }^{34}$ The temperature modulation depends on the following parameters

$$
\Delta T=\gamma P_{\text {pump }} f\left[R_{\text {ring }}, \omega(\lambda, z)\right] R_{t h} \stackrel{\approx \alpha\left(\lambda_{\text {pump }}\right) L_{\text {eff }}}{\left(1-e^{-\alpha\left(\lambda_{\text {pump }}\right) L_{\text {eff }}}\right)}
$$

with $\gamma$ the thermal power yield which is the fraction of the absorbed power that is converted to heat. $(1-\gamma)$ is the fraction of the absorbed power re-emitted through luminescence processes. ${ }^{17}$ Luminescent yield is notoriously known to be very low in the mid-infrared and therefore $\gamma$ is assumed to be constant over the measured mid-infrared wavelength range and equal to unity ${ }^{17,35}$. $P_{\text {pump }}$ is the power exiting the $\mathrm{ZrF}_{4}$ fiber, $f$ is the fraction of the optical power of a Gaussian beam contained within a radius $R_{\text {ring }}$ for a beam with waist $\omega(\lambda, z)$ where $z$ is the distance of the fiber facet to the ring resonator, $R_{t h}$ is the effective thermal resistance of the transducer, $\alpha$ is the absorption coefficient of the analyte and $L_{e f f}$ is the effective thickness of the analyte.

The reported variation of the Mode-Field Diameter (MFD) of the SM mid-infrared fiber in the measured wavelength range is taken into account for the calculation of $f .{ }^{36}$ The resulting function $f$ increases $\sim 20 \%$ in absolute value from $3250 \mathrm{~nm}$ to $3600 \mathrm{~nm}$. Typical parameters used for the experiment are listed in table 1. 
Table 1: Parameters used to calculate the photothermal absorption spectrum and some typical values for the two measurements from Figure 4. An error estimate is given where applicable.

\begin{tabular}{|c|c|c|c|c|}
\hline parameter & $\mathrm{I}$ & II & error & unit \\
\hline$P_{\text {probe,max }}{ }^{a}$ & -32.3 & -28.8 & 0.1 & $\mathrm{dBm}$ \\
\hline$|d \mathcal{T} / d \lambda|_{\max }^{b}$ & 122 & 63 & $10 \%$ & $\mathrm{~nm}^{-1}$ \\
\hline$Q^{b}$ & $166 \mathrm{k}$ & $77 \mathrm{k}$ & $10 \%$ & \\
\hline$\lambda_{r e s}{ }^{a}$ & 1550.076 & 1549.232 & 0.001 & $\mathrm{~nm}$ \\
\hline$P_{\text {pump }}\left(2921.4 \mathrm{~cm}^{-1}\right)^{a}$ & 0.45 & 0.35 & 0.04 & $\mathrm{~mW}$ \\
\hline$\Delta T\left(2921.4 \mathrm{~cm}^{-1}\right)$ & 11.0 & 9.8 & $20 \%$ & $\mathrm{mK}$ \\
\hline$\alpha\left(2921.4 \mathrm{~cm}^{-1}\right)$ & 745.2 & 646.6 & $25 \%$ & $\mathrm{~cm}^{-1}$ \\
\hline$f\left[R_{\text {ring }}\right.$ & & & & \\
\hline$\left.\omega\left(2921.4 \mathrm{~cm}^{-1}, 100 \mu \mathrm{m}\right)\right]^{c}$ & 0.86 & 0.64 & $10 \%$ & \\
\hline$R_{t h}^{c}$ & 142 & 273 & $1 \%$ & $\mathrm{~K} / \mathrm{W}$ \\
\hline$R_{\text {ring }}$ & 35 & 25 & & $\mu m$ \\
\hline resolution & 5 & 20 & & $\mathrm{~nm}$ \\
\hline total time & 35 & 10 & & $\min$ \\
\hline$z$ & \multicolumn{2}{|c|}{100} & 10 & $\mu m$ \\
\hline$G$ & \multicolumn{2}{|c|}{$10^{6}$} & & $\mathrm{~V} / \mathrm{W}$ \\
\hline$n_{g}{ }^{b}$ & \multicolumn{2}{|c|}{3.71} & $5 \%$ & \\
\hline$\partial n / \partial T$ & \multicolumn{2}{|c|}{$7.6 \times 10^{-4}$} & & $K^{-1}$ \\
\hline$L_{e f f}$ & \multicolumn{2}{|c|}{2.8} & 0.01 & $\mu m$ \\
\hline integration time & \multicolumn{2}{|c|}{100} & & $\mathrm{~ms}$ \\
\hline$f_{m o d}$ & \multicolumn{2}{|c|}{300} & & $\mathrm{~Hz}$ \\
\hline
\end{tabular}




\section{IRRAS}

To evaluate the photothermal spectroscopic method, the absorption spectrum of the analyte was evaluated using Infrared Reflection Absorption Spectroscopy (IRRAS) using TMpolarized light and under the Brewster angle of the analyte/air interface. A Varian 680 Fourier Transform Infrared Spectrometer (FTIR) was used. The incidence angle was adjusted using the Veemax accesory from Pike to the Brewster angle of 58 degrees for the polymer/air interface. A ZnSe wire grid polarizer is used to obtain TM polarization. In this configuration, the specular reflection from the air/polymer interface is eliminated such that only the beam reflected off of the silicon substrate is collected. The sample is therefore measured in transflectance mode ${ }^{37,38}$ the light effectively traverses the sample twice, see also the inset of Figure 4. A $1.35 \mu \mathrm{m}$ thick sample was prepared by spincoating the AZ5214 photoresist onto a silicon wafer. The absorption spectrum is estimated by assuming the Beer-Lambert law and comparing the reflectance spectrum $R_{s}(\lambda)$ to the reflectance $R_{0}(\lambda)$ of a plain silicon wafer under identical conditions, see also the equation in Figure 4 . The absorption coefficient $\alpha$ is on the order of $0.05 \mu m^{-1}$.

\section{Results and discussion}

Figure 4 shows the measured photothermal spectrum with the absorption spectrum calculated using equations 1 and 2 and the comparison with the FTIR spectrum. The peak positions $\left(3016.7,2921.4\right.$ and $\left.2862.9 \mathrm{~cm}^{-1}\right)$ corresponding to the aliphatic $\mathrm{C}-\mathrm{H}$ stretching vibration frequencies agree well with the results obtained from IRRAS. ${ }^{39}$

The magnitude of the measured absorption coefficient also corresponds quite well with the IRRAS results. Figure 4 also shows that two different devices measured under different conditions produce the same absorption spectrum by normalizing the signal to the measurable quantities in table 1. This shows that as long as the position of the pump fiber to the chip is reasonably known and controlled, the variability of on-chip devices (e.g. Q-factor) can be 


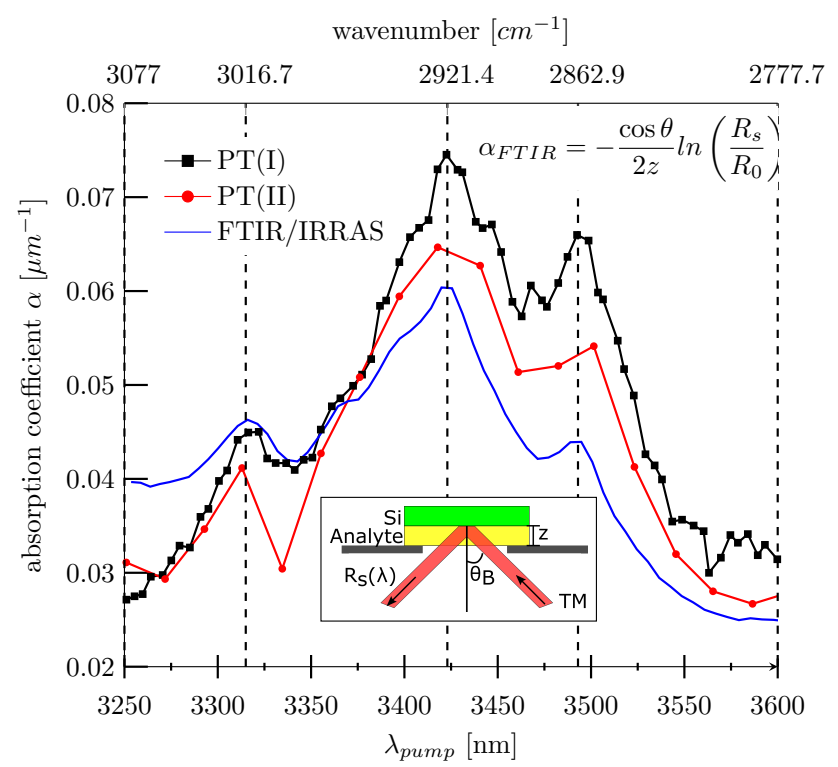

Figure 4: Comparison of the absorption spectrum of the photoresist AZ5214 recovered by the photothermal method (PT) for two different devices under different measurement conditions I and II (see table 1) to the absorption spectrum measured with the FTIR. The PT absorption coefficient is estimated using equations 1 and 2. For the FTIR measurement, the inset shows that the absorption coefficient $\alpha_{F T I R}$ is estimated by comparing the reflectance spectrum of the analyte to the reflectance spectrum of silicon (see formula in graph). Measurements are performed under the Brewster angle (58 degrees) and TM polarization to eliminate fringes of the transflectance spectrum. 
accounted for. An estimate of the error on the parameters used for the calculation of the absorption is given in table 1. A safe value of $10 \%$ was assumed for the fitting algorithm to retrieve the Q-factor and derivative from the measurement. By considering the worst case scenario for the error addition in the calculation, it is estimated that $\alpha$ at $2921 \mathrm{~cm}^{-1}$ for experiment II in Figure 4 lies within the extreme bounds of $500-870 \mathrm{~cm}^{-1}$.

Even after taking the beam waist wavelength dependency into account, there are minor discrepancies in the relative peak heights of the photothermally recovered spectrum and the FTIR spectrum. This is due to the fact that the photothermal technique probes the nonradiative absorption directly whereas the FTIR technique is influenced by optical scattering effects. ${ }^{17}$ It is well known that optical scattering is stronger for shorter wavelengths and this could explain why the FTIR technique overestimates the nonradiative absorption at shorter wavelengths.

The device acts as a wavelength-selective bolometer for the mid-infrared source. The sensitivity $S_{\text {max }} / P_{\text {pump }}$ is estimated in Figure 5 and equals $150 \mathrm{~V} / \mathrm{W}$ at the peak absorption wavelength of the polymer of $2921.4 \mathrm{~cm}^{-1}$ with $\alpha_{2921.4 \mathrm{~cm}^{-1}} \approx 750 \mathrm{~cm}^{-1}$ for a non-suspended resonator. The probe power $P_{\text {probe, max }}$ for the measurement is $1.6 \mu \mathrm{W}$. Further improvements can be achieved by thermally isolating the ring resonators.

In the current setup, the measurement time is on the order of 10 minutes. The scanning time of the experiments can be greatly reduced by considering a different mid-infrared source and reducing the measured wavelength range. An External Cavity-Quantum Cascade Laser (EC-QCL) can be used which can achieve $\mathrm{kHz}$ scanning rates in a $\sim 7 \mathrm{~cm}^{-1}$ wavelength range or $20-40 \mathrm{~Hz}$ rates in $\mathrm{a} \approx 100-150 \mathrm{~cm}^{-1}$ wavelength range. ${ }^{40,41}$

\section{Thermal isolation}

Thermal isolation of the ring resonators can greatly increase the photothermal signal response. Thermal steady-state FEM simulations were performed using COMSOL. The effec- 


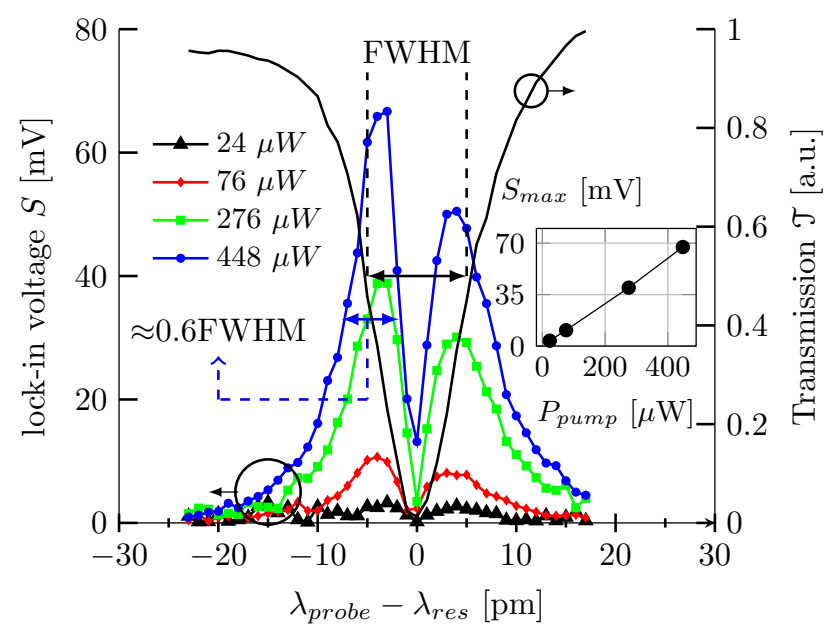

Figure 5: Photothermal signal on the lock-in amplifier for increasing $P_{\text {pump }}$ corresponding to measurement $I$ in Figure 4 at $\lambda_{\text {pump }}=2921.4 \mathrm{~cm}^{-1}$. $P_{\text {pump }}$ is the power exiting the midinfrared fiber facet towards the resonator. The device can be viewed as a thermal detector for the mid-infrared source with a sensitivity $S / P_{\text {pump }}$ of $150 \mathrm{~V} / \mathrm{W}$ for $P_{\text {probe }} \approx 1.6 \mu W$. The FWHM of the optical resonator is $10 \mathrm{pm}$. The FWHM of the photothermal signal is $\approx 0.6 \times$ smaller.

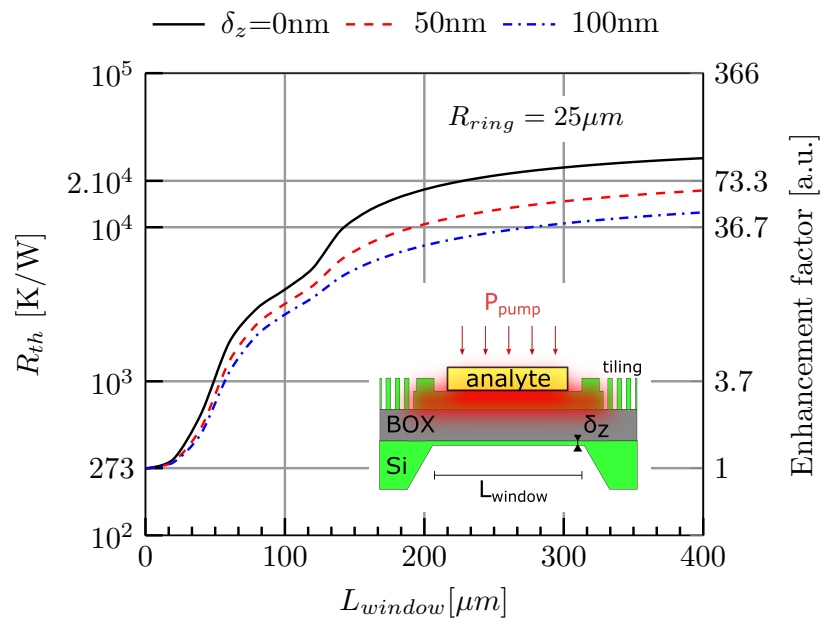

Figure 6: 3D-axial symmetrical FEM simulation of the effective thermal resistance of suspended ring resonators. The expected increase in thermal resistance $R_{t h}$ for a window of $280 \times 280 \mu m^{2}$ is on the order of $\times 100$. A thin, sub-micron thick $\delta_{z}$ remaining silicon slab on the backside of the membrane can significantly reduce the isolation factor. The influence of the tiling (indicated in blue in Figure $2(\mathrm{c})$ ) has been accounted for by adding concentric $\mathrm{Si}$ rings with a period of $3 \mu \mathrm{m}$ and $70 \%$ fill factor on top of the BOX. The value for $L_{\text {window }}=0$ corresponds to experiment I in Figure 4 
tive thermal resistance $R_{t h}$ was estimated by calculating the average temperature increase in the ring waveguide for a given power dissipation.

In Figure 6, the results of a 3D axial symmetrical model are given with the axis of symmetry in the center of the ring and normal to the surface. As seen in the microscope and SEM images in Figure 2, the top silicon slab is only continuous in a square $120 \times 120 \mu m^{2}$ area. Beyond this slab, the top layer is filled with tiling, which thermally disconnects the top silicon layer. This tiling layer is included in the simulation by adding a periodically etched slab beyond the $60 \mu \mathrm{m}$ radius with a period of $3 \mu \mathrm{m}$ and a fill factor of $70 \%$, hereby modeling the tiling as concentric rings. From Figure 6 it is clear that care has to be taken that the silicon substrate is etched completely up to the BOX for maximum effectiveness. The heat dissipation to the silicon substrate is the dominant heat loss mechanism. From the microscope and SEM images in Figure 2, one should also consider that (a) the opened window is unintentionally shifted by $25 \mu \mathrm{m}$ with respect to the center of the ring and (b) the ring resonator area is thermally connected to the rest of the silicon slab on the device layer by $\approx 5 \mu m$ wide strips along the access waveguides, which contribute to the heat dissipation to the sides (indicated in red in Figure 2(c)). Full 3D simulations (without the tiling layer) indicate a decrease of the enhancement factor by $10 \%$ to account for (a) and an additional $20 \%$ decrease to account for (b). For $L_{\text {window }}=280 \mu m$, the expected enhancement factor from Figure 6 is corrected to $\approx 57$ for better correspondence with the structure depicted in Figure 2 .

The fabricated membrane ring resonators were compared to identical devices on silicon substrate with the same measurement setup. The acquired maximal lock-in signal $S$ for a certain $\lambda_{\text {pump }}$ is normalized to the reference pump power $P_{\text {pump }}$ and $P_{\text {probe }}$, see Figure 7 . In order to evaluate the photothermal enhancement, differences between the maximal slopes of the two ring resonators are taken into account by normalizing the maximal photothermal response to the corresponding maximal slope $|d \mathcal{T} / d \lambda|_{\text {max }}$. The slopes are extracted from the 
Lorentzian transmission from Figure S2 in the Supporting Information. The performance ratios of the suspended resonators as compared to the supported ones are given in table 2.

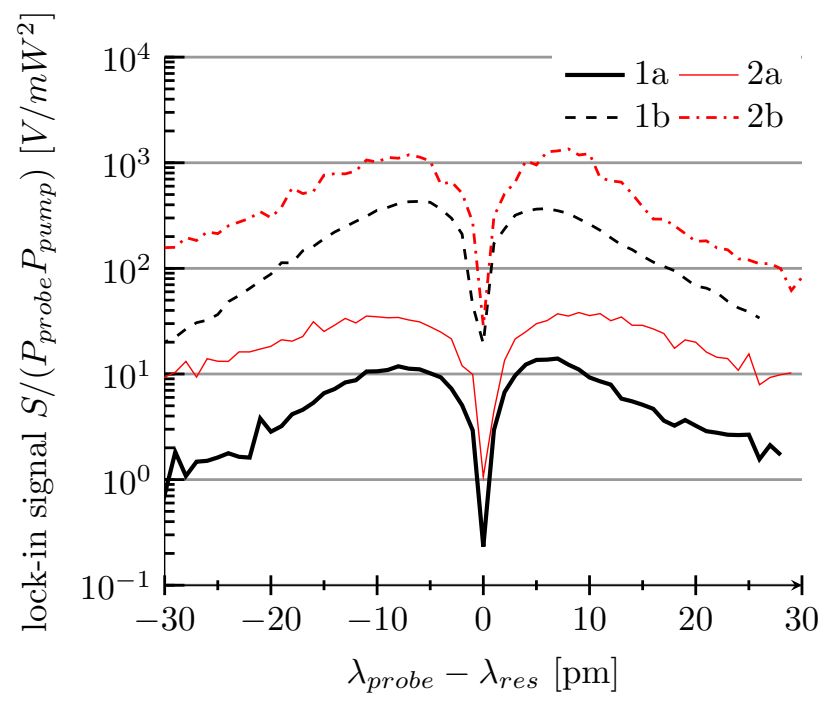

Figure 7: Normalized photothermal signal modulation $S$ of $1.35 \mu m$ thick AZ5214 photoresist at $\lambda_{\text {pump }}=3419 \mathrm{~nm}\left(2925 \mathrm{~cm}^{-1}\right)$ for (a) supported and (b) suspended ring resonators. Two different ring resonators were compared. The maximum photothermal signal is enhanced by more than $10 \mathrm{~dB}$ in both cases.

Table 2: Performance comparison of suspended (B) resonators to supported ones (A). Quantities are expressed as ratios $(\mathrm{B} / \mathrm{A})$.

\begin{tabular}{lll}
\hline device & 1 & 2 \\
\hline $\mathcal{T}_{\text {probe }, B} / \mathcal{T}_{\text {probe }, A}$ & 1.014 & 1.445 \\
$\left|\frac{d \mathfrak{T}}{d \lambda}\right|_{\text {max }, B} /\left|\frac{d \mathfrak{T}}{d \lambda}\right|_{\text {max }, A}$ & 0.72 & 1.0 \\
{$\left[S_{\text {max }} / P_{\text {probe }} P_{\text {pump }}\right]_{B} /\left[S_{\text {max }} / P_{\text {probe }} P_{\text {pump }}\right]_{A}$} & 30.8 & 34.5 \\
$R_{\text {th }, B} / R_{\text {th }, A}$ & 43.1 & 34.5 \\
\hline
\end{tabular}

The best measured enhancement factor $E=R_{t h, B} / R_{t h, A}$ of 43 is slightly lower than what is expected from simulations.

\section{Limit of Detection}

It is clear that a high slope of the transmission spectrum is beneficial for the photothermal signal and thus a high Q-factor resonator is desirable. Ultra high-Q disk resonators have 
been shown. ${ }^{42}$ However, from a practical point of view, it becomes very difficult to lock the probe wavelength to the optimal operating point. As indicated in Figure 5, the Full Width at Half-Maximum (FWHM) of the photothermal signal is $\approx 0.6$ times smaller than the FWHM of the Lorentzian resonance transmission. For a ring resonance with $Q>300 k$ this gives a photothermal FWHM $<3$ pm, approaching the limits of the accuracy of typical external cavity lasers. Therefore we believe the presented ring resonators with Q factors around 100k are a good trade-off between sensitivity and practival feasibility.

The limit of detection (LOD) is evaluated for the thermally isolated device (1b), as shown in Figure 2 and whose measurement results were previously shown in Figure 7. The RMS deviation of the lock-in signal $S$ (as defined in equation 1) at the optimal probe position is $0.49 \mathrm{mV}$ for a $100 \mathrm{~ms}$ integration time, $P_{\text {probe } \max }=0.5 \mu \mathrm{W}$ and gain $G=10^{6} \mathrm{~V} / \mathrm{W}$. This corresponds to a minimal detectable change in probe transmission of $\Delta \mathcal{T}_{\text {probe }}=5 \times 10^{-4}$, which is a reasonable estimate for lock-in type detection schemes. Given the increased thermal resistance of approximately $R_{t h} \sim 1.2 \times 10^{4} \mathrm{~K} / \mathrm{W}$ this leads to an estimated normalized noise equivalent absorption coefficient $\left(\mathrm{NNEA}=\alpha_{\min } P_{\text {pump }} / \sqrt{\Delta f}\right)$ of $7.6 \times 10^{-6} \mathrm{~cm}^{-1} \mathrm{~W} / \mathrm{Hz} z^{1 / 2}$ for $P_{\text {probe } \max }=0.5 \mu \mathrm{W}$. The on-chip probe power cannot be chosen too large as the thermal non-linear effects would affect the response of the ring resonator. With a fiber power $P_{\text {pump }}$ of $50 \mathrm{~mW}, P_{\text {probe,on-chip }}=0.5 \mu \mathrm{W}$ and 1s integration time, a minimal detectable absorption coefficient $\alpha_{\min }$ of $1.5 \times 10^{-4} \mathrm{~cm}^{-1}$ is predicted.

Instead of the photoresist demonstrated here, a gas-adsorbing porous coating with preconcentration factors as high as $10^{4}$ can be patterned for practical trace gas sensing applications. ${ }^{4,5,43}$ Typical absorption values of trace gases in the 3-4 $4 m$ wavelength region are of the order of $30 \mathrm{~cm}^{-1}$ at 1 atm and room temperature. ${ }^{44}$ Even without assuming gas preconcentration inside the coating, this gives a LOD estimate of $c_{\min }=5 \mathrm{ppm}$. 
The performance in terms of NNEA reported here is modest compared to state-of-the-art minuaturized optical sensors such as some QEPAS examples where NNEA values as low as $10^{-10} \mathrm{~cm}^{-1} \mathrm{~W} / \mathrm{Hz}^{1 / 2}$ have been shown. ${ }^{28,29}$ However the proposed transducer shows that non-contact all-optical interrogation is possible which can be a key requirement in certain applications, e.g. sensors incorporated in food packages. Additionally, the use of a preconcentrating coating is expected to bring down the NNEA to competitive values.

For real-life applications such as trace gas detection in food packages, it would be advisable to select a narrower wavelength range of $\approx 100-150 \mathrm{~cm}^{-1}$ to target only a limited number of absorbing species. This way, a more compact, faster and relatively cheaper mid-infrared source can be used such as a Distributed Feedback (DFB) QCL or EC-QCL. Furthermore, free-space optics for the probe and pump beam can be used, as opposed to fibers, to flood illuminate the chip from a short ( $<1$ meter) distance. As only the ring can be illuminated with the mid-IR beam, an optical aperture can be defined on top of the ring resonator sensor, that is aligned with the ring resonator, reflecting or absorbing all other pump light, see also Figure S5 in the Supporting Information. A collimated pump with a millimeter sized beam diameter would have a fraction of the power contained on the resonator on the order of $10^{-3}$, a loss which could be compensated by the coating pre-concentration factor. Small position variations of the beam would then not affect the average pump power density delivered to the coating. The transmitted probe signal could then be collected by a near-IR camera ${ }^{5}$.

\section{Conclusions}

An on-chip mid-IR photothermal spectroscopic sensor using suspended silicon-on-insulator microring resonators is demonstrated. The transducer circumvents the need of using a midinfrared detector by transducing the heat generated through optical absorption to a cheaper near-infrared read-out system. The sensor was used to recover the absorption spectrum of the photoresist AZ5214 in the 3.25-3.6 $\mu \mathrm{m}$ wavelength range in good agreement with bench- 
mark FTIR techniques.

The photothermal signal has been increased by a factor of 40 by opening a window in the silicon substrate beneath the resonator, hereby suspending the ring resonators on the buried oxide and thus thermally isolating the ring waveguide. The resulting effective thermal resistance is estimated to be $1.2 \times 10^{4} \mathrm{~K} / \mathrm{W}$. The latter results in a NNEA of $7.6 \times 10^{-6} \mathrm{~cm}^{-1} \mathrm{~W} / \mathrm{Hz}^{1 / 2}$ for $0.5 \mu W$ of probe power. By using a pump power of $50 \mathrm{~mW}$ and 1 s integration time, a LOD $\alpha_{\min }$ of $1.5 \times 10^{-4} \mathrm{~cm}^{-1}$ is predicted. Thermal FEM simulations suggest that further improvements by a factor of 2-3 can be made by making sure that the ring resonator is completely thermally disconnected from the rest of the chip on the device layer. This can be achieved by etching trenches in the device layer close to the ring waveguide.

For practical trace gas sensing applications, a gas-adsorbing porous coating can be patterned on the ring transducers. Sub-ppm LOD values are expected for trace gases absorbing in the $3-4 \mu m$ wavelength range.

This sensing approach is specifically interesting for all-optical non-contact trace gas detection applications. For example, the disposable and cheap SOI ring resonator transducer can be embedded inside a food package, whereas the read-out unit containing the tunable probe and pump sources are positioned at some distance above the package. This photothermal spectroscopy approach would then allow, e.g. for a selective and sensitive way of detecting trace gases originating from spoilage without the need of using a cooled mid-infrared detector.

Supporting Information Available: The following files are available free of charge.

- Supporting Information: optical properties of the suspended membranes; FEM simulation of stress due to mechanical shock; time evolution of the optimal probe wavelength during measurement; schematic of a possible free-space measurement configuration. 


\section{Acknowledgement}

The author thanks the Research Foundation Flanders (FWO) for a research grant and the CheckPack IWT project funded by the Flemish government.

\section{References}

(1) Soref, R. The Past, Present, and Future of Silicon Photonics. IEEE J. Sel. Top. Quantum Electron. 2006, 12, 1678-1687.

(2) Vos, K. D.; Bartolozzi, I.; Schacht, E.; Bienstman, P.; Baets, R. Silicon-on-Insulator microring resonator for sensitive and label-free biosensing. Opt. Express 2007, 15, 76107615.

(3) Nitkowski, A.; Chen, L.; Lipson, M. Cavity-enhanced on-chip absorption spectroscopy using microring resonators. Opt. Express 2008, 16, 11930-11936.

(4) Yebo, N. A.; Lommens, P.; Hens, Z.; Baets, R. An integrated optic ethanol vapor sensor based on a silicon-on-insulator microring resonator coated with a porous $\mathrm{ZnO}$ film. Opt. Express 2010, 18, 11859-11866.

(5) Yebo, N. A.; Sree, S. P.; Levrau, E.; Detavernier, C.; Hens, Z.; Martens, J. A.; Baets, R. Selective and reversible ammonia gas detection with nanoporous film functionalized silicon photonic micro-ring resonator. Opt. Express 2012, 20, 11855-11862.

(6) Muneeb, M.; Vasiliev, A.; Ruocco, A.; Malik, A.; Chen, H.; Nedeljkovic, M.; Penades, J. S.; Cerutti, L.; Rodriguez, J. B.; Mashanovich, G. Z.; Smit, M. K.; Tourni, E.; Roelkens, G. III-V-on-silicon integrated micro - spectrometer for the $3 \mu \mathrm{m}$ wavelength range. Opt. Express 2016, 24, 9465-9472.

(7) Zlatanovic, S.; Park, J. S.; Moro, S.; Boggio, J. M. C.; Divliansky, I. B.; Alic, N.; Mookherjea, S.; Radic, S. Mid-infrared wavelength conversion in silicon waveguides 
using ultracompact telecom-band-derived pump source. Nat. Photonics 2010, 4, 561564 .

(8) Wang, R.; Muneeb, M.; Sprengel, S.; Boehm, G.; Malik, A.; Baets, R.; Amann, M.-C.; Roelkens, G. III-V-on-silicon 2- $\mu$ m-wavelength-range wavelength demultiplexers with heterogeneously integrated InP-based type-II photodetectors. Opt. Express 2016, 24, 8480-8490.

(9) Roelkens, G. et al. Silicon-based heterogeneous photonic integrated circuits for the mid-infrared. Opt. Mater. Express 2013, 3, 1523-1536.

(10) Spott, A.; Peters, J.; Davenport, M. L.; Stanton, E. J.; Merritt, C. D.; Bewley, W. W.; Vurgaftman, I.; Kim, C. S.; Meyer, J. R.; Kirch, J.; Mawst, L. J.; Botez, D.; Bowers, J. E. Quantum cascade laser on silicon. Optica 2016, 3, 545-551.

(11) Mashanovich, G. Z. et al. Silicon and germanium mid-infrared photonics. Proc. SPIE 2016, 9755, 97550W:1-97550W:6.

(12) Soref, R. Mid-infrared photonics in silicon and germanium. Nat. Photonics 2010, 4, $495-497$.

(13) Chen, Y.; Lin, H.; Hu, J.; Li, M. Heterogeneously Integrated Silicon Photonics for the Mid-Infrared and Spectroscopic Sensing. ACS Nano 2014, 8, 6955-6961.

(14) Lin, H.; Li, L.; Zou, Y.; Danto, S.; Musgraves, J. D.; Richardson, K.; Kozacik, S.; Murakowski, M.; Prather, D.; Lin, P. T.; Singh, V.; Agarwal, A.; Kimerling, L. C.; Hu, J. Demonstration of high-Q mid-infrared chalcogenide glass-on-silicon resonators. Opt. Lett. 2013, 38, 1470-1472.

(15) Li, L.; Lin, H.; Qiao, S.; Zou, Y.; Danto, S.; Richardson, K.; Musgraves, J. D.; Lu, N.; $\mathrm{Hu}$, J. Integrated flexible chalcogenide glass photonic devices. Nat. Photonics 2014, 8, 643-649. 
(16) Han, Z.; Lin, P.; Singh, V.; Kimerling, L.; Hu, J.; Richardson, K.; Agarwal, A.; Tan, D. T. H. On-chip mid-infrared gas detection using chalcogenide glass waveguide. Appl. Phys. Lett. 2016, 108, 141106:1-141106:3.

(17) Bialkowski, S. Photothermal spectroscopy methods for chemical analysis; John Wiley \& Sons, 1996; Vol. 134.

(18) Hu, J. Ultra-sensitive chemical vapor detection using micro-cavity photothermal spectroscopy. Opt. Express 2010, 18, 22174-22186.

(19) Gaiduk, A.; Yorulmaz, M.; Ruijgrok, P. V.; Orrit, M. Room-Temperature Detection of a Single Molecule's Absorption by Photothermal Contrast. Science 2010, 330, 353-356.

(20) Yamada, S.; Schmid, S.; Larsen, T.; Hansen, O.; Boisen, A. Photothermal Infrared Spectroscopy of Airborne Samples with Mechanical String Resonators. Anal. Chem. 2013, 85, 10531-10535.

(21) Stievater, T. H.; Papanicolaou, N. A.; Bass, R.; Rabinovich, W. S.; McGill, R. A. Micromechanical photothermal spectroscopy of trace gases using functionalized polymers. Opt. Lett. 2012, 37, 2328-2330.

(22) Wei Jin, F. Y., Yingchun Cao; Ho, H. L. Ultra-sensitive all-fibre photothermal spectroscopy with large dynamic range. Nat. Commun. 2015, 6, 6767:1-6767:8.

(23) Yang, F.; Tan, Y.; Jin, W.; Lin, Y.; Qi, Y.; Ho, H. L. Hollow-core fiber Fabry-Perot photothermal gas sensor. Opt. Lett. 2016, 41, 3025-3028.

(24) Heylman, K. D.; Knapper, K. A.; Goldsmith, R. H. Photothermal Microscopy of Nonluminescent Single Particles Enabled by Optical Microresonators. J. Phys. Chem. Lett. 2014, 5, 1917-1923.

(25) Vermeulen, P.; Cognet, L.; Lounis, B. Photothermal microscopy: optical detection of small absorbers in scattering environments. J. Microsc. 2014, 254, 115-121. 
(26) Nedosekin, D. A.; Galanzha, E. I.; Dervishi, E.; Biris, A. S.; Zharov, V. P. SuperResolution Nonlinear Photothermal Microscopy. Small 2014, 10, 135-142.

(27) Ding, T. X.; Hou, L.; van der Meer, H.; Alivisatos, A. P.; Orrit, M. Hundreds-fold Sensitivity Enhancement of Photothermal Microscopy in Near-Critical Xenon. J. Phys. Chem. Lett. 2016, 7, 2524-2529.

(28) Spagnolo, V.; Patimisco, P.; Borri, S.; Scamarcio, G.; Bernacki, B. E.; Kriesel, J. Partper-trillion level SF 6 detection using a quartz enhanced photoacoustic spectroscopybased sensor with single-mode fiber-coupled quantum cascade laser excitation. Opt. Lett. 2012, 37, 4461-4463.

(29) Patimisco, P.; Scamarcio, G.; Tittel, F. K.; Spagnolo, V. Quartz-Enhanced Photoacoustic Spectroscopy: A Review. Sensors 2014, 14, 6165-6206.

(30) imec, Europractice Homepage. 2016; http://www.europractice-ic.com.

(31) Lang, B. W. Structural Dynamics, Volume 3; Springer, 2011; pp 955-960.

(32) Morrison, A. M.; Liang, T.; Douberly, G. E. Automation of an Aculight continuouswave optical parametric oscillator. Rev. Sci. Instrum. 2013, 84, 013102:1-013102:8.

(33) Bogaerts, W.; De Heyn, P.; Van Vaerenbergh, T.; De Vos, K.; Kumar Selvaraja, S.; Claes, T.; Dumon, P.; Bienstman, P.; Van Thourhout, D.; Baets, R. Silicon microring resonators. Laser Photonics Rev. 2012, 6, 47-73.

(34) Wong, C. Y.; Cheng, Z.; Chen, X.; Xu, K.; Fung, C. K.; Chen, Y. M.; Tsang, H. K. Characterization of mid-infrared silicon-on-sapphire microring resonators with thermal tuning. IEEE Photonics J. 2012, 4, 1095-1102.

(35) Klan, P.; Wirz, J. Photochemistry of organic compounds: From concepts to practice; John Wiley \& Sons, 2009. 
(36) Thorlabs, MIR Single Mode Fluoride Fiber Optic Patch Cables. 2016; https://www. thorlabs.de/newgrouppage9.cfm?objectgroup_id=7999.

(37) Peter R. Griffiths, J. A. D. H.; Winefordner, J. D. Fourier Transform Infrared Spectrometry, 2nd Edition; John Wiley \& Sons, 2007.

(38) Teolis, B.; Loeffler, M.; Raut, U.; Fama, M.; Baragiola, R. Infrared reflectance spectroscopy on thin films: Interference effects. Icarus 2007, 190, $274-279$.

(39) Larkin, P. Infrared and Raman Spectroscopy; Principles and Spectral Interpretation; Elsevier, 2011.

(40) Tsai, T.; Wysocki, G. External-cavity quantum cascade lasers with fast wavelength scanning. Appl. Phys. B 2010, 100, 243-251.

(41) Wysocki, G.; Lewicki, R.; Curl, R.; Tittel, F.; Diehl, L.; Capasso, F.; Troccoli, M.; Hofler, G.; Bour, D.; Corzine, S. Widely tunable mode-hop free external cavity quantum cascade lasers for high resolution spectroscopy and chemical sensing. Appl. Phys. B 2008, 92, 305-311.

(42) D. K. Armani, S. M. S., T. J. Kippenberg; Vahala, K. J. Ultra-high-Q toroid microcavity on a chip. Nature 2003, 421, $925-928$.

(43) Xu, P.; Yu, H.; Li, X. Functionalized mesoporous silica for microgravimetric sensing of trace chemical vapors. Anal. Chem. 2011, 83, 3448-3454.

(44) Workman Jr, J. The Handbook of Organic Compounds, Three-Volume Set: NIR, IR, R, and UV-Vis Spectra Featuring Polymers and Surfactants; Elsevier, 2000. 


\section{Graphical TOC Entry}

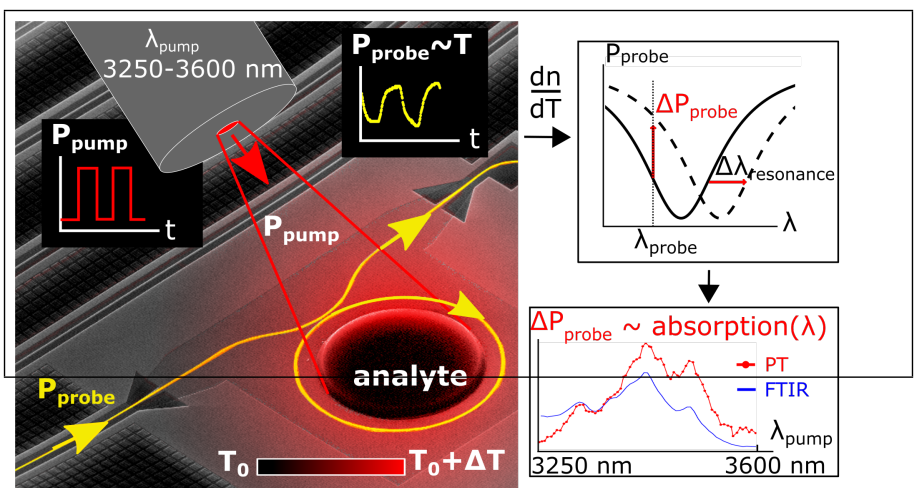

